\title{
Social Determinants of Polypharmacy in First Generation Mexican Immigrants in the United States
}

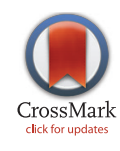

\author{
Shervin Assari ${ }^{1 *}$, Mohammed Saqib ${ }^{2}$, Cheryl Wisseh ${ }^{1,3}$, Mohsen Bazargan ${ }^{1,4}$ \\ ${ }^{1}$ Department of Family Medicine, Charles R. Drew University of Medicine and Science, Los Angeles, USA \\ ${ }^{2}$ Health Behavior and Health Education (HBHE), University of Michigan, Ann Arbor, MI, USA \\ ${ }^{3}$ Department of Pharmacy Practice, West Coast University School of Pharmacy, Los Angeles, CA, USA \\ ${ }^{4}$ Department of Family Medicine, UCLA, Los Angeles, USA
}

Corresponding Author: Shervin Assari, MD, MPH, Department of Family Medicine, Charles R. Drew University of Medicine and Science, Los Angeles, USA. Tel: +1-734-232-0445, Fax: +1-734-615-8739, Email: assari@umich.edu

Received April 10, 2019; Accepted August 4, 2019; Online Published September 14, 2019

\begin{abstract}
Introduction: Socioeconomic status (SES) indicators are among the main social determinants of health and illness. Less, however, is known about the role of SES in the epidemiology of polypharmacy in immigrant Latino Americans living in the United States. This research studied the association between three SES indicators, education, income, and employment, and polypharmacy in older first generation Latino American immigrant adults.

Methods: Data was obtained from the Sacramento Area Latino Study on Aging (SALSA, 1996-2008). A total of 632 older first generation Mexican-American immigrants to the U.S. entered this analysis. The independent variables were education, income, and employment. Polypharmacy was the outcome. Age, gender, physical health, smoking, and drinking were the covariates. Binary logistic regression was used to analyze the data.

Results: Employment was associated with lower odds of polypharmacy. The association between education and polypharmacy was above and beyond demographic factors, physical health, health behaviors, and health insurance. Neither education nor income were associated with polypharmacy. Other determinants of polypharmacy were poor self-rated health (SRH) and a higher number of chronic medical conditions (CMCs).

Conclusion: Employment appears to be the major SES determinant of polypharmacy in older foreign-born Mexican Americans. Unemployed older Mexican American immigrants with multiple chronic diseases and those who have poor SRH have the highest need for an evaluation of polypharmacy. Given the age group of this population, most of them have health insurance, which provides an opportunity for reducing their polypharmacy.

Keywords: Educational Achievement, Income, Mexican American, Immigrants, Ethnic Groups, Polypharmacy
\end{abstract}

Citation: Assari S, Saqib M, Wisseh C, Bazargan M. Social determinants of polypharmacy in first generation Mexican immigrants in the United States. Int J Travel Med Glob Health. 2019;7(3):86-90. doi:10.15171/ijtmgh.2019.19.

\section{Introduction}

Given the various undesired health outcomes associated with polypharmacy, ${ }^{1}$ there is an interest in epidemiological studies that determine the distribution of polypharmacy in society. ${ }^{2}$ These epidemiological studies highlight the sub-sections of the society and population that are most strongly affected by polypharmacy.,3 Based on the observations of these studies, similar to many other health outcomes, polypharmacy is not distributed at random, but it is more common in the sections of society with the greatest social and health needs. ${ }^{3,4}$

Very little is known about the social determinants of polypharmacy in racial and ethnic minority populations. ${ }^{5}$ Across various racial and ethnic minorities in the U.S., more research is done on social determinants concerning African Americans than Latinos. ${ }^{5,6}$ As a result, there is a need for studies on the association between social determinants of health (SDOH) and polypharmacy among Latino Americans, particularly each subgroup of Latino Americans. As Latino immigrants to the U.S. are composed of diverse and heterogenous groups that vary in country of origin, nativity, and immigration status, studies that explore the link between $\mathrm{SDOH}$ and polypharmacy in each specific group of Latino Americans based on such characteristics are needed. The current study focuses on first generation immigrants to the United States and not on those born to parents who immigrated.

Copyright $\odot 2019$ The Author(s). This is an open-access article distributed under the terms of the Creative Commons Attribution License (http:// creativecommons.org/licenses/by/4.0), which permits unrestricted use, distribution, and reproduction in any medium, provided the original work is properly cited. 
There are some inconsistencies regarding the association between $\mathrm{SDOH}$ and polypharmacy in the United States. While some studies show that high SES reduces the prevalence of polypharmacy, ${ }^{5}$ other studies have suggested opposite results. ${ }^{6}$ This inconsistency is because on one hand, high SES is associated with lower health needs and chronic medical conditions (CMCs), ${ }^{5}$ and on the other hand, high SES may be a gateway for people to use services, be diagnosed, and be prescribed. ${ }^{6}$ Such associations in opposite directions may cancel the effects of one other or cause inconsistencies across settings, populations, age groups, and SES indicators. This is particularly dependent on the population and country, as correlations between health and health needs vary from one setting $^{7-10}$ or population ${ }^{8,11}$ to another.

To extend the existing knowledge on the link between $\mathrm{SDOH}$ and polypharmacy, the current study investigated the effects of three SES indicators (namely education, income, and employment) on polypharmacy among older Mexican American adults. All participants were born in Mexico and had immigrated to the U.S. at some time in their lives. This study hypothesized that among older immigrant Mexican Americans, at least one of the SES indicators, either high education, income, or employment, would be associated with lower odds of polypharmacy. No hypothesis on which SES would be a predictor of polypharmacy was made.

\begin{abstract}
Methods
Design and Setting

The Sacramento Area Latino Study on Aging (SALSA, 19962008) was a longitudinal cohort study of older adults with Latino ethnicity in Sacramento county and neighboring counties. Sampling, design, measuring, and rationale have previously been extensively described. ${ }^{12-18}$ The data collected included rich information on demographics, socioeconomic status (SES), medication use, health, and cultural factors. Overall, the baseline SALSA study comprised a total of 1,789 Latinos age $60+$ years. While initial sampling and recruitment occurred between 1998 and 1999, participants were under follow-up evaluations through 2007. Follow-up visits and observations were conducted every $12-15$ months. ${ }^{15,17}$
\end{abstract}

\section{Sample, Sampling Strategy, and Sample Size}

The SALSA study used a random sampling of community dwelling Latino Americans. Participants were recruited from counties with proportional densities of Latinos of at least 5\% based on information from the 1990 census. Approximately $22 \%$ of the total eligible population of Sacramento County, CA, was recruited to the SALSA study. From the total sample of 1,789 Latino Americans, 632 older first generation Mexican American adult immigrants to the U.S. had data on variables in the current study (analytical sample size).

\section{Inclusion and Exclusion Criteria}

Participants were eligible if they: (1) self-identified as Latino or Latino, (2) were age 65+ years, (3) were born in Mexico, (4) had immigrated to the U.S. some time in their lives, and (5) lived in the U.S. during their older adult life. Exclusion criteria was living in a institutionalized setting. The study did not exclude individuals based on language, so both Spanish and English speakers were enrolled.

\section{Outcome}

This study defined polypharmacy as the use of $5+$ medications in a single day. This definition was based on a Systematic review of definitions of polypharmacy across studies. Although there is no consensus, this is probably the most common definition of polypharmacy used by researchers. ${ }^{1}$ In this study, polypharmacy was calculated based on a comprehensive evaluation of all medications that each individual was taking.

\section{Independent Variables}

Education, income, and employment were the primary independent variables of interest in this study. All three variables were measured as self-reported data. Education was defined as years of formal education and schooling. Household annual income was also self-reported. Education and income were continuous measures. Employment was a dichotomous variable: $1=$ employed, $0=$ not in the labor market or unemployed.

\section{Covariates}

Retirement, language, self-rated health (SRH), number of CMCs, smoking, and drinking were covariates in the current study. Smoking and drinking were dichotomous variables. Smoking was 1 for current smoker, and 0 for never or past smoker. Drinking was 1 for those who reported they drink. $\mathrm{SRH}$ was the conventional single item, ranging from 1 to 5 , with a higher score indicating worse health. The item read, "Would you say, in general, your health is: 1) Excellent, 2) Very good, 3) Good, 4) Fair, or 5) Poor?” Self-reported language proficiency was another covariate. Participants rated how well they spoke English and Spanish on a four-point scale: 0 - not at all, 1 - a little, 2 - well, 3 - very well. The use of English versus Spanish was coded if either language was spoken "very often," "almost always," "not very often," or "not at all."

\section{Statistics}

SPSS version 23.0 was used for data analysis. Frequencies, means, and standard deviations (SDs) were reported for descriptive purposes. A binary logistic regression model was run for multivariable analysis. In this model, polypharmacy was the outcome; educational attainment, income, and employment were the predictors of interest. "Enter" selection of the variables in the regression model was used. Thus, the variables remained in the model regardless of their significance. Demographics, health behaviors, physical health, and health insurance were the covariates. Odds ratio (OR), 95\% confidence interval (CI), and $P$ value were reported.

\section{Results}

Univariate Analysis

A total number of 632 participants entered the study. Table 1 shows a summary of the descriptive statistics for the sample. All individuals were at least 65 years old, and average age among participants was 74 years. In the sample, $56.5 \%$ were 
Table 1. Descriptive Characteristics of Participants

\begin{tabular}{|c|c|c|}
\hline & No. & $\%$ \\
\hline \multicolumn{3}{|l|}{ Gender } \\
\hline Female & 351 & 56.5 \\
\hline Male & 270 & 43.5 \\
\hline \multicolumn{3}{|l|}{ Employment } \\
\hline No & 535 & 89.5 \\
\hline Yes & 63 & 10.5 \\
\hline \multicolumn{3}{|l|}{ Insurance } \\
\hline No & 52 & 8.2 \\
\hline Yes & 580 & 91.8 \\
\hline \multicolumn{3}{|l|}{ Smoking (current) } \\
\hline No & 558 & 88.6 \\
\hline Yes & 72 & 11.4 \\
\hline \multicolumn{3}{|l|}{ Drinking } \\
\hline No & 333 & 52.7 \\
\hline Yes & 299 & 47.3 \\
\hline \multicolumn{3}{|l|}{ Polypharmacy } \\
\hline No & 534 & 84.5 \\
\hline \multirow[t]{2}{*}{ Yes } & 98 & 15.5 \\
\hline & Mean & SD \\
\hline Age (y) & 73.95 & 6.81 \\
\hline Household income & 1.71 & 1.07 \\
\hline Education (y) & 4.38 & 4.34 \\
\hline Spanish language (1-4) & 2.85 & 0.48 \\
\hline $\mathrm{CMC}(\mathrm{n})$ & 1.63 & 1.46 \\
\hline SRH (1-5) & 0.59 & 0.49 \\
\hline ADL & 0.97 & 3.09 \\
\hline
\end{tabular}

Abbreviations: CMCs, chronic medical conditions; ADL, activities of daily living; SRH: self-rated health.

women. From all participants, only $8.2 \%$ did not have health insurance, $10.5 \%$ were working at the time of survey, and $15.5 \%$ had polypharmacy.

\section{Multivariable Analysis}

Table 2 shows the results of a logistic regression model. This model included SES as well as demographic factors, physical health, and health behaviors. As this model shows, the main determinants of polypharmacy were number of CMCs (OR $=1.52,95 \% \mathrm{CI}=1.30-1.78, P=0.000)$ and having poor $\mathrm{SRH}$ $(\mathrm{OR}=2.54,95 \% \mathrm{CI}=1.41-4.57, \quad P=0.002)$. In addition, employment was associated with odds of polypharmacy (OR $=0.21,0.05-0.95, P=0.042)$. Each additional CMC would increase the odds of polypharmacy by $52 \%$. Employment would be associated with a $79 \%$ reduction in the odds of polypharmacy. Poor/fair SRH was associated with a $154 \%$ increase in the odds of polypharmacy.

\section{Discussion}

The current study aimed to study social determinants of polypharmacy in older Latino Americans who had immigrated from Mexico to the U.S. A binary logistic regression model was applied to data from the Sacramento Area Latino Study to test the association between three key SES indicators, namely education, income, and employment, with polypharmacy. It was found that employment status was associated with lower odds of polypharmacy in this population. This association was above and beyond demographic factors, physical health,
Table 2. Logistic Regression on Polypharmacy in Older Foreign-Born Mexican American Adults

\begin{tabular}{llll}
\hline & OR & $\mathbf{9 5} \% \mathbf{C l}$ & $\boldsymbol{P}$ Value \\
\hline Age & 0.98 & $0.94-1.02$ & 0.276 \\
Gender (male) & 0.67 & $0.39-1.15$ & 0.144 \\
Household income & 1.15 & $0.89-1.49$ & 0.296 \\
Education (y) & 0.97 & $0.91-1.04$ & 0.414 \\
Employed & 0.21 & $0.05-0.95$ & 0.042 \\
Health insurance & 5.72 & $0.75-43.63$ & 0.092 \\
Spanish language (1-4) & 0.79 & $0.48-1.31$ & 0.363 \\
CMC (n) & 1.52 & $1.30-1.78$ & 0.000 \\
SRH (poor/fair) & 2.54 & $1.41-4.57$ & 0.002 \\
ADL & 1.03 & $0.95-1.13$ & 0.467 \\
Smoking (current) & 1.42 & $0.66-3.07$ & 0.369 \\
\hline Drinking & 0.94 & $0.55-1.58$ & 0.805 \\
Constant & 0.09 & & 0.236 \\
\hline
\end{tabular}

Abbreviations: CMCs, chronic medical conditions; ADL, activities of daily living; SRH: self-rated health.

health behaviors, and health insurance.

One of the mechanisms by which SES indicators impact health is through better access to health care services. Individuals with high SES have better access to health care; thus, they are more likely to use preventive services, diagnostic services, and receive a better quality of treatment when needed. Countries, however, differ in the type of SES indicators. In the United States, for example, employment is the gateway to the health care system. Thus, Americans who are unemployed may be at an increased risk for poor health outcomes, in part due to lack of health care coverage. This study, however, showed an effect of employment, while the effect of health care coverage was controlled. This suggests that the health effect of employment is not only through access to the health care system.

Multiple studies across various cultures, countries, and settings have investigated the association between various SES indicators and polypharmacy. ${ }^{4}$ In some settings, income, ${ }^{5,19}$ and in other settings, education ${ }^{5}$ may be the SES indicator that impacts the prevalence of polypharmacy. The links between various SES indicators and polypharmacy are shown in China, ${ }^{19}$ Sweden, ${ }^{4}$ and the United States, ${ }^{5}$ among other countries. The literature showed that different SES indicators are relevant to polypharmacy across different settings. However, research is very limited concerning foreign-born Mexican American older adults. The current findings suggest that in the U.S. and for older foreign-born Mexican American adults, employment status, and not education or income, correlates with polypharmacy.

Various attempts have been made to reduce polypharmacy, including de-prescribing movements and initiatives. ${ }^{20}$ Other protocols include spending more time reviewing the medications that a patient takes. It is shown that even a 10-minute discussion with patients can make a difference and reduce the rate of polypharmacy. ${ }^{21}$ Some of these measures have promising effects on increasing adherence, simply 
because polypharmacy reduces adherence. ${ }^{20}$ These efforts can be made by physicians ${ }^{20}$ or pharmacists. ${ }^{22}$ Review articles have shown that mnemonics, algorithms, clinical practice guidelines, and clinical strategies have been studied as a tool to address polypharmacy in a variety of health care settings. ${ }^{23}$ Modest effects of interventions are shown for educational programs that help doctors reduce polypharmacy. At the same time, more assertive interventions that directly caution physicians to avoid polypharmacy are promising. ${ }^{24}$

More research is needed on ways by which polypharmacy can be reduced in immigrants, particularly when they are in their senior stages of life. There is a paucity of literature on culturally appropriate and effective methods to reduce polypharmacy among Latino immigrants residing in the U.S. Even the mainstream literature has several limitations, including the lack of adequate control variables, low sample sizes, poor measurements, duration of follow up, poor randomization, and inadequate monitoring of potential adverse effects. More research is needed to overcome these methodological limitations. ${ }^{25}$ More research is also needed to understand how SES disparities in polypharmacy in nonimmigrants as well as immigrants can be reduced.

Studies have shown that income $e^{5,19}$ and education ${ }^{5}$ impact the prevalence of polypharmacy. Similar patterns have been observed in various age groups, including children, ${ }^{3}$ adults, ${ }^{3}$ and older adults. ${ }^{5}$ Some research suggests that there is a trend in increasing polypharmacy among low SES individuals. ${ }^{3}$ Very few studies have been conducted on Latino and Mexican immigrants. Thus, the current study makes a unique contribution to the field as it extends the literature from the mainstream population to older Mexican Americans who, at one point in their lives, migrated to the U.S.

This study focused on Mexican Americans who were born outside the US. It has shown that the effect of SES on polypharmacy varies across ethnic groups. ${ }^{5}$ For example, one study showed that low education attainment and low income predicted higher odds of polypharmacy in African American older adults. That study, however, showed a stronger social gradient in polypharmacy in African Americans and further showed that low income might be a more strong predictor of polypharmacy in African Americans than Whites. ${ }^{5}$ More research is needed on first- and second- generation older adults who have migrated from other countries to the U.S. It might be that, for Mexican American immigrants, employment becomes the main SES driver of polypharmacy.

\section{Limitations}

No study is without methodological limitations. One limitation of the current study was the cross-sectional design. It is known that SES and health have bidirectional effects. While low SES predicts low health, health is also needed for maintaining social status. Thus, no causal association can be evidenced from observations of a cross-sectional association between education and polypharmacy. Health insurance was an unmeasured confounder and should be included in any future research on this topic. Wealth and other SES indicators were also not considered. Another limitation was that this study included only Mexican Americans. Other ethnic groups

\section{Research Highlights}

\section{What Is Already Known?}

In some previous studies, socioeconomic status has been linked to polypharmacy. Very little information, however, is found on the same pattern in older foreign-born Mexican Americans.

\section{What This Study Adds?}

This study adds to the literature that low socioeconomic status is associated with high polypharmacy in older foreign-born Mexican Americans. Between various SES indicators, employment seems to show an effect, while education and income do not show strong effects. Unemployed foreign-born older Mexican Americans who have multiple chronic conditions should be assessed for inappropriate polypharmacy.

as well as Latinos who are immigrants from other countries should be investigated. Despite these limitations, this study makes a contribution and extends the existing knowledge on social determinants of polypharmacy in ethnic groups in the U.S.

\section{Conclusion}

It seems that high SES is associated with reduced odds of polypharmacy in older foreign-born Mexican American adults. Between education, income, and employment, the latter seems to be the major SES determinant of polypharmacy in older Mexican American adults who migrated to the U.S. at some point in their lives.

\section{Authors' Contributions}

Conceptualization, SA; Data curation, SA; Formal analysis, SA; Funding acquisition, MB; Writing - original draft, SA; Writing - review and editing, SA, MS, CW, and MB.

\section{Conflict of Interest Disclosures}

The authors declare no conflicts of interest.

\section{Ethical Approval}

All participants signed a written consent form. The SALSA protocol was reviewed and approved by the Institutional Review Boards of the University of California (UC), Davis and the University of Michigan (UM).

\section{Funding/Support}

This study was supported by the Center for Medicare and Medicaid Services (CMS) Grant 1H0CMS331621 (PI: Bazargan). Assari and Bazargan are also supported by National Institutes of Health (NIH) grants D084526-03, CA201415 02, DA035811-05, U54MD008149, U54MD007598, and U54CA229974.

\section{References}

1. Leelakanok N, Holcombe AL, Lund BC, Gu X, Schweizer ML. Association between polypharmacy and death: A systematic review and meta-analysis. J Am Pharm Assoc (2003). 2017;57(6):729738.e710. doi:10.1016/j.japh.2017.06.002. 
2. Armstrong KS, Temmingh $\mathrm{H}$. Prevalence of and factors associated with antipsychotic polypharmacy in patients with serious mental illness: Findings from a cross-sectional study in an uppermiddle-income country. Braz J Psychiatry. 2017;39(4):293-301. doi:10.1590/1516-4446-2016-2015.

3. Baek YH, Shin JY. Trends in polypharmacy over 12 years and changes in its social gradients in South Korea. PLoS One. 2018;13(9):e0204018. doi:10.1371/journal.pone.0204018.

4. Haider SI, Johnell K, Thorslund M, Fastbom J. Analysis of the association between polypharmacy and socioeconomic position among elderly aged $>$ or $=77$ years in Sweden. Clin Ther. 2008;30(2):419-427. doi:10.1016/j.clinthera.2008.02.010.

5. Assari S, Bazargan M. Race/Ethnicity, Socioeconomic Status, and Polypharmacy among Older Americans. Pharmacy (Basel). 2019;7(2). doi:10.3390/pharmacy7020041.

6. Assari S, Helmi H, Bazargan M. Polypharmacy in African American Adults: A National Epidemiological Study. Pharmacy (Basel). 2019;7(2). doi:10.3390/pharmacy7020033.

7. Assari S, Moghani Lankarani M. Does multi-morbidity mediate the effect of socioeconomics on self-rated health? Cross-country differences. Int J Prev Med. 2015;6:85. doi:10.4103/20087802.164413 .

8. Assari S. Cross-country variation in additive effects of socioeconomics, health behaviors, and comorbidities on subjective health of patients with diabetes. J Diabetes Metab Disord. 2014;13(1):36. doi:10.1186/2251-6581-13-36.

9. Assari S, Moghani Lankarani R, Moghani Lankarani M. Crosscountry differences in the association between diabetes and disability. J Diabetes Metab Disord. 2014;13(1):3. doi:10.1186/2251-6581-13-3.

10. Assari S. Cross-country differences in the additive effects of socioeconomics, health behaviors and medical comorbidities on disability among older adults with heart disease. J Tehran Heart Cent. 2015;10(1):24-33.

11. Assari S. Combined racial and gender differences in the longterm predictive role of education on depressive symptoms and chronic medical conditions. J Racial Ethn Health Disparities. 2017;4(3):385-396. doi:10.1007/s40615-016-0239-7.

12. Zeki Al Hazzouri A, Haan MN, OsypukT, Abdou C, Hinton L, Aiello AE. Neighborhood socioeconomic context and cognitive decline among older Mexican Americans: results from the Sacramento Area Latino Study on Aging. Am J Epidemiol. 2011;174(4):423431. doi:10.1093/aje/kwr095.

13. Haan MN, Zeki Al-Hazzouri A, Aiello AE. Life-span socioeconomic trajectory, nativity, and cognitive aging in Mexican Americans: the Sacramento Area Latino Study on Aging. J Gerontol B Psychol Sci Soc Sci. 2011;66 Suppl 1:i102-110. doi:10.1093/geronb/gbq071.

14. Zeki Al Hazzouri A, Haan MN, Kalbfleisch JD, Galea S, Lisabeth LD, Aiello AE. Life-course socioeconomic position and incidence of dementia and cognitive impairment without dementia in older
Mexican Americans: results from the Sacramento area Latino study on aging. Am J Epidemiol. 2011;173(10):1148-1158. doi:10.1093/ aje/kwq483.

15. Garcia L, Lee A, Zeki Al Hazzouri A, Neuhaus J, Epstein M Haan M. The impact of neighborhood socioeconomic position on prevalence of diabetes and pre-diabetes in older Latinos: the Sacramento area Latino study on aging. Hisp Health Care Int. 2015;13(2):77-85. doi:10.1891/1540-4153.13.2.77.

16. Afable-Munsuz A, Mayeda ER, Pérez-Stable EJ, Haan MN. Immigrant generation and diabetes risk among Mexican Americans: the Sacramento Area Latino Study on Aging. Am J Public Health. 2013;103(5):e45-52. doi:10.2105/AJPH.2012.300969.

17. Rodriquez EJ, Sabado-Liwag M, Pérez-Stable EJ, et al. Allostatic Load, Unhealthy Behaviors, and Depressive Symptoms by Birthplace Among Older Adults in the Sacramento Area Latino Study on Aging (SALSA). J Aging Health. 2019:898264319857995. doi:10.1177/0898264319857995.

18. González HM, Haan MN, Hinton L. Acculturation and the prevalence of depression in older Mexican Americans: baseline results of the Sacramento Area Latino Study on Aging. J Am Geriatr Soc. 2001;49(7):948-953. doi:10.1046/j.15325415.2001.49186.x.

19. Xiang YT, Weng YZ, Leung CM, Tang WK, Ungvari GS. Clinical and social determinants of antipsychotic polypharmacy for Chinese patients with schizophrenia. Pharmacopsychiatry. 2007;40(2):4752. doi:10.1055/s-2007-970062.

20. Ulley J, Harrop D, Ali A, Alton S, Fowler Davis S. Deprescribing interventions and their impact on medication adherence in community-dwelling older adults with polypharmacy: a systematic review. BMC Geriatr. 2019;19(1):15. doi:10.1186/s12877-0191031-4.

21. Walsh EK, Cussen K. "Take ten minutes": a dedicated ten minute medication review reduces polypharmacy in the elderly. Ir Med J. 2010;103(8):236-238.

22. van der Meer HG, Wouters H, Pont LG, Taxis K. Reducing the anticholinergic and sedative load in older patients on polypharmacy by pharmacist-led medication review: a randomised controlled trial. BMJ Open. 2018;8(7):e019042. doi:10.1136/ bmjopen-2017-019042.

23. Skinner M. A literature review: polypharmacy protocol for primary care. Geriatr Nurs. 2015;36(5):367-371.e364. doi:10.1016/j. gerinurse.2015.05.003

24. Tani H, Uchida H, Suzuki T, Fujii Y, Mimura M. Interventions to reduce antipsychotic polypharmacy: a systematic review. Schizophr Res. 2013;143(1):215-220. doi:10.1016/j.schres.2012.10.015.

25. Tranulis C, Skalli L, Lalonde P, Nicole L, Stip E. Benefits and risks of antipsychotic polypharmacy: an evidence-based review of the literature. Drug Saf. 2008;31(1):7-20. doi:10.2165/00002018200831010-00002. 